FORMATION Formation emploi

Revue française de sciences sociales

124 | Octobre-Décembre 2013

Pêle-mêle

\title{
La mesure des tutorats en entreprise : enjeux, complexité et limites
}

La mesure des tutorats en entreprise : enjeux, complexité et limites

The measure of Mentoring tasks in companies: stakes, complexity and limits

Ausbildung im Betrieb: Herausforderungen, Vielschichtigkeit und Grenzen

La medición de las tutorías en empresa: desafíos, complejidad y límites

Catherine Delgoulet, Alexandre Largier et Ghislaine Tirilly

\section{OpenEdition}

Journals

Édition électronique

URL : http://journals.openedition.org/formationemploi/4079

DOI : 10.4000/formationemploi.4079

ISSN : 2107-0946

Éditeur

La Documentation française

Édition imprimée

Date de publication : 31 décembre 2013

Pagination : 45-62

ISSN : 0759-6340

Référence électronique

Catherine Delgoulet, Alexandre Largier et Ghislaine Tirilly, « La mesure des tutorats en entreprise enjeux, complexité et limites », Formation emploi [En ligne], 124 | Octobre-Décembre 2013, mis en ligne le 08 janvier 2015, consulté le 10 décembre 2020. URL : http://journals.openedition.org/ formationemploi/4079; DOI : https://doi.org/10.4000/formationemploi.4079 


\title{
La mesure des tutorats en entreprise : enjeux, complexité et limites
}

\author{
Catherine Delgoulet \\ Ergonome, maître de conférences au Laboratoire Adaptations Travail - Individus (LATI) de \\ I'université Paris Descartes, Sorbonne Paris Cité \\ AleXANDRE LARGIER \\ Sociologue, SNCF, Direction Cohésion et Ressources Humaines \\ GHISLAINE TIRILLY \\ Ergonome, SNCF, Direction Cohésion et Ressources Humaines
}

Résumé

\section{La mesure des tutorats en entreprise : enjeux, complexité et limites}

La conjoncture sociale et économique réactualise le tutorat en entreprise. Cet article propose une réflexion sur ce mode d'acquisition et de transfert des savoirs ou savoir-faire et sa mesure à partir de deux études réalisées auprès d'agents de maintenance et de gestionnaires de paie, au sein d'une grande entreprise de service. Les analyses confirment le caractère protéiforme du tutorat et permettent d'identifier quelques raisons qui rendent difficile sa mesure. Elles invitent à préférer à la mesure une démarche d'évaluation intégrée (quantitative/qualitative).

Mots clés : tutorat, entreprise, évaluation

Abstract

The measure of Mentoring tasks in companies: stakes, complexity and limits

Social and economical developments update the mentoring tasks in companies. The aim of this paper is to think about this acquisition way and knowledge or abilities changeover and his measure from two field studies relevant to maintenance operators and pay manager in a big service company. Analyses confirm the protean characteristics of mentoring and allow identifying some reasons, which get some difficulties to measure its cost. They invite to complete the quantitative measure with a qualitative component.

Keywords: tutoring, company, evaluation

Journal of Economic Literature: M 53, J 24

Traduction : Auteurs 
Le tutorat d'entreprise est depuis de nombreuses années au cœur d'enjeux importants dans le monde du travail (Boru \& Leborgne, 1992 ; Barbier, 1996 ; Fredy-Planchot, 2007). Son développement dans le cadre de dispositifs de formation en alternance ou du Plan national d'action concerté pour l'emploi des seniors (Conseil économique et social, 2006) qui retient le tutorat comme l'un des cinq objectifs de promotion du maintien et du retour en emploi des seniors, confirme son actualité.

Au sein de la grande entreprise du secteur des transports partenaire de cette réflexion, le tutorat, entendu au sens large, est une forme d'acquisition et de transfert des savoirs et savoir-faire utilisée en parallèle ou en complément d'autres dispositifs de formation professionnelle. Les nouvelles obligations juridiques (liées au Plan national d'action pour l'emploi des seniors), la configuration démographique de la population des salariés de l'entreprise ${ }^{1}$, mais aussi la volonté de réduire des dépenses de formation parfois jugées peu utiles parce que non directement productives, ont amené l'entreprise à requestionner le dispositif du tutorat. L'hypothèse sous-jacente à cette demande étant que ce dispositif de formation serait plus souple et moins onéreux que les formations professionnelles plus classiques. Pour cela, une étude menée au sein de l'entreprise a visé à cartographier les diverses formes de tutorat existantes et à analyser, sous l'angle économique, les coûts et les gains qu'elles génèrent ${ }^{2}$ (Reyre et al., 2011). Si la phase de cartographie n'a pas posé de problème majeur, la phase d'évaluation économique a surtout permis de souligner la difficulté de prendre la mesure du tutorat. Ce constat est ici l'occasion de revenir sur la question en tentant d'identifier quelques conditions de la pertinence et du cadre de la mesure.

Pour cela, nous présenterons tout d'abord les formes de tutorat qui se développent dans l'entreprise, avant d'expliciter les raisons qui rendent difficiles le calcul des coûts et des gains inhérents au tutorat. En discussion et conclusion, des éléments de réponse aux questions de la mesure seront développés. À chaque étape, l'argumentation s'appuiera sur des travaux d'étude et de recherche menés dans cette entreprise ; bien que n'étant pas à l'origine de la demande, la question du tutorat traversait les analyses réalisées

1. Il s'agit d'une population vieillissante, dont les départs massifs en cours et à venir laissent entrevoir des pertes de compétences dans certaines activités (Jolivet et al., 2009).

2. Cette étude repose d'une part sur une analyse des documents définissant et cadrant les diverses formes de tutorat à l'œuvre dans l'entreprise et, d'autre part, sur 36 entretiens et 6 journées d'observation ethnographique sur trois sites volontaires (en établissement d'accueil clientèle, dans un atelier climatisation, et dans une brigade de signalisation électrique). En outre, une méthodologie particulière, dite "instruction au sosie ", a été utilisée pour faciliter une posture réflexive des tuteurs sur leur activité (Kloetzer, 2011). Elle procède d'une expérience de substitution fictive de rôle. A l'occasion d'un entretien, l'interviewé reçoit pour consigne de transmettre à l'interviewer (le sosie) les recommandations nécessaires pour que ce dernier soit en mesure de le remplacer dans son travail au quotidien sans que personne ne se rende compte de la substitution. Ceci permet d'interroger une activité spécifique, ici de tutorat, pour la retravailler (pour en savoir plus, $c f$. Oddone, 1981 ; Clot, 2001). 
(cf. encadré 1). Dans ce cadre, deux populations contrastées du point de vue des tâches qu'elles accomplissent et des conditions de travail ont participé aux investigations : d'une part, les agents de maintenance des installations, population exclusivement masculine, chargés de l'entretien et du remplacement de certaines parties des installations ; d'autre part, les gestionnaires de paie, population majoritairement féminine, qui gèrent les dossiers administratifs des salariés de l'entreprise (rémunération, mobilité, absences de toute nature, etc.).

\section{Le tutorat : un dispositif protéiforme et contingent}

Laccent est mis ici sur les diverses formes que peuvent prendre les situations tutorales rencontrées - malgré l'usage du terme générique " tutorat » - et leur dépendance aux conditions matérielles et organisationnelles de travail dans lesquelles elles s'inscrivent.

\subsection{Un dispositif au caractère protéiforme}

Le tutorat peut être défini " comme une approche pédagogique fondée sur une situation de travail accompagnée [et un] outil de socialisation professionnelle" (Fredy-Planchot, 2007, p. 24). Le tutorat remplit donc à la fois une fonction de transmission, d'échange de compétences et une fonction d'accueil, d'intégration des nouvelles recrues. La littérature signale également que ce dispositif de formation peut être guidé par des buts d'accompagnement des changements (techniques, organisationnels, sociaux) dans le travail ou de développement des compétences des tuteurs dans une perspective à moyen terme de mobilité professionnelle de cette population (Barbier, 1996 ; Wittorski, 1996).

L'étude des modalités du tutorat de l'entreprise, citée précédemment (Reyre et al., 2011), confirme le caractère protéiforme de ce dispositif de formation, comme ont pu le souligner les travaux précédemment cités. En reprenant la proposition de Wittorski (1996) de distinguer le tutorat de "reproduction de pratiques" du tutorat "d'anticipation des changements", l'analyse documentaire des guides ou fiches de tutorat (lorsque ceux-ci existent), les observations en situation de travail réalisées dans le cadre de cette étude et les entretiens menés auprès de divers acteurs (les tuteurs eux-mêmes, les novices, l'encadrement de proximité, $c f$. encadré 1 ), permettent de différencier quatre formes de tutorat, considérées comme idéales-typiques de ce qui est réellement mis en place dans l'entreprise. La catégorisation proposée pour outiller les analyses distingue les formes de tutorat en fonction de l'objectif qu'elles poursuivent (la reproduction ou l'adaptation aux évolutions) et de l'organisation réelle de l'activité tutorale (en binôme ou collective ; $c$. tableau $\mathbf{1}$ ). 


\section{Encadré 1}

\section{Méthodologie}

L'ensemble de nos données repose sur une recherche menée au sein d'une grande entreprise française de service, entre 2010 et 2011.

Cette recherche consiste à évaluer les effets des changements socio-organisationnels et managériaux sur les collectifs de travail et la santé de leurs membres (Largier et Tirilly, 2011). Les grandes thématiques abordées sont les dimensions collectives du travail, les modalités de réalisation du travail, l'activité, les changements organisationnels et les questions liées au parcours et à la formation. Les questions liées au tutorat étaient particulièrement sensibles dans les collectifs qui, suite à certains changements, connaissaient un turnover trés important. Cette recherche repose sur deux études de cas : (1) les équipes de gestionnaires de paie et (2) les équipes de la maintenance des installations.

La première étude a été réalisée au sein du pôle de gestionnaires de paie (PGP) des directions centrales qui gère les dossiers des salariés des directions transverses. Ce PGP est constitué d'une équipe de direction de 14 personnes et de 56 gestionnaires, majoritairement des femmes. Trois des six équipes de ce PGP se sont portées volontaires pour participer à l'étude (soit 30 personnes).

La majeure partie de l'activité de ces gestionnaires est consacrée au calcul des salaires (« la solde »). Depuis sa création, en 2008, ce PGP a subi de nombreuses transformations socio-organisationnelles et managériales dont les principales conséquences sont une recomposition de l'ensemble des acteurs des ressources humaines (la « ligne $\mathrm{RH}$ » - ressources humaines -, selon les acteurs) et une intensification du travail. Une des motivations à la création du PGP était de spécialiser certains gestionnaires des anciens bureaux administratifs dans la réalisation de la solde. En les spécialisant, le nombre de dossiers de salariés en portefeuille a augmenté et il a fallu recruter un nombre important de salariés. Par conséquent, la formation et l'intégration des nouveaux salariés s'ajoutent à l'augmentation du nombre de dossiers de salariés en portefeuille et à la recomposition de la « ligne $\mathrm{RH} »$, pesant ainsi sur les conditions de réalisation de l'activité.

La deuxième étude concerne des agents de maintenance, soumis à de nombreuses évolutions structurelles (l'extension des territoires d'intervention, fusions et scissions d'établissements, etc.) ayant des répercussions sur leurs effectifs et les modalités de réalisation de leur activité. Ainsi, l'encadrement de proximité a vu sa charge de travail s'accroître fortement avec l'éloignement géographique entre les équipes et leurs hiérarchies. Les agents de maintenance ont pour missions la surveillance, le diagnostic et l'entretien (réparation, remplacement) des installations sur un territoire défini ainsi que la mise en sécurité des zones de chantier, pour eux-mêmes ou d'autres intervenants. Il s'agit d'une activité qui nécessite une forte coopération.

Nos analyses s'appuient sur 46 entretiens semi-directifs, menés auprès d'agents de maintenance (29 entretiens) et de gestionnaires volontaires ( 17 entretiens), ainsi que sur plusieurs journées d'immersion et d'observation de l'activité (sur des chantiers de maintenance et dans les open-spaces du PGP). Les acteurs rencontrés ont tous été, ou bien sont, encadrés par un tuteur à leur arrivée dans la fonction. De même, les salariés aujourd'hui expérimentés se trouvent ou se sont trouvés en position de participer à au moins une situation tutorale formelle ou informelle. Les entretiens ont été analysés à travers des matrices thématiques reprenant les grands thèmes énoncés plus haut (dimensions collectives du travail, modalités de réalisation de l'activité, changements organisationnels et parcours et formation, Miles \& Huberman, 2003). Les journées d'immersion et d'observation nous ont permis de mieux appréhender la réalité des situations de travail dans lesquelles tuteurs et apprenants évoluent, de manière à affiner ensuite le guide d'entretien. 


\section{Tableau 1}

Quatre formes idéales-typiques de tutorat dans l'entreprise de transport

\begin{tabular}{|l|c|c|}
\hline & Reproduction & Adaptation aux évolutions \\
\hline Binôme & $\begin{array}{c}\text { Compagnonnage (équipes de mainte- } \\
\text { nance matériel) }\end{array}$ & Binômage (équipes de vente) \\
\hline Collectif & $\begin{array}{c}\text { Tutorat collégial (équipes de maintenance } \\
\text { des installations) }\end{array}$ & Tutorat tournant (équipes de gestionnaires) \\
\hline
\end{tabular}

Source : auteurs.

Comme le suggère Wittorski (1996), ces deux premières dimensions sont à croiser, d'une part, avec le caractère formel ou informel des tutorats ; d'autre part, avec les conditions matérielles et organisationnelles qui conditionnent les possibilités réelles de déploiement des projets de reproduction ou d'adaptation aux évolutions du travail. L'ensemble de ces dimensions multiplie une fois encore les possibilités de configurations, sans avoir la prétention d'en épuiser les modalités.

\subsection{Le tutorat : une activité collective, en binôme ou en équipe}

Au-delà de l'objectif affiché, attendu implicitement ou détourné, de l'activité tutorale, les conditions de travail et modalités d'organisation prescrites ou effectives facilitent ou au contraire bousculent les intentions initiales du tutorat. Barbier (1996) pointe la « contingence du tutorat " par rapport aux situations réelles de travail qui, par leur caractère répétitif, mais aussi changeant ou insolite, conditionnent le développement et la consolidation des compétences professionnelles des apprenants. Wittorski (1996) évoque quant à lui le rôle des conditions de travail des tuteurs comme potentialités de leur propre développement. Ces éléments semblent essentiels à l'efficience du dispositif de tutorat mais leur déclinaison en situation demeure parfois problématique.

Dans l'entreprise étudiée, un grand nombre de formes de tutorat met aux prises un tuteur et un apprenant. C'est bien sûr officiellement le cas des situations d'apprentissage (formule existante mais qui ne s'est pas présentée lors de nos investigations), mais cela concerne aussi les binômes mis en place dans certains services de gestion de la paie pour former de nouvelles recrues. On constate, par ailleurs, que lorsqu'il est formalisé, le tutorat est toujours envisagé selon cette modalité, à l'image du compagnonnage. Cependant, les conditions de transmission (la durée, la reconnaissance de cette activité, la relation d'autorité entre tuteur et apprenti, etc.) ne sont pas comparables à celles historiquement instituées. On remarque que même lorsqu'un binôme est formellement constitué, la nouvelle recrue reçoit des informations, des conseils, d'un grand nombre d'acteurs : "A chaque fois qu'un agent nouveau arrive dans l'équipe, il y a un relais à faire, [...] Bénédicte , je l'ai mise

3. Par respect de l'anonymat, les prénoms des salariés ont tous été changés. 
avec Fabienne, qui était très structurée et c'est ce dont Bénédicte avait besoin. Nathalie a été en formation avec Ophélie. Dans la réalité des faits Henri, Zohra et d'autres y ont participé. » (Michèle, 50 ans, manager de proximité, équipe de gestionnaires de paie, 32 ans d'expérience en gestion administrative)

Dans d'autres cas, l'activité tutorale est plus largement et ouvertement collective, même si rien n'est explicitement formalisé. Ce sont plutôt les aléas de la production ou de la mission de service, les imprévus des conditions de réalisation des tâches qui " imposent " à une équipe de prendre en charge l'intégration de la nouvelle recrue et la transmission des connaissances utiles (Gaudart et al., 2008). Ceci s'observe par exemple au sein de certaines équipes de salariés de maintenance : "Comment on m'a expliqué le métier? Je montais avec eux et ils m'expliquaient. [...] Quand jétais à $N$., ceétait avec tout le monde. [Et à $M$ ?] Avec tout le monde aussi. C'est à $N$. que j'ai commencé à monter. On me montrait ce qu'il fallait faire et ce qu'il ne fallait pas faire. On me donnait les filons. " (Jonathan, 21 ans, 3 ans d'ancienneté et d'expérience en maintenance des installations). Parce que différents chantiers sont réalisés en parallèle, que les congés et les périodes de formation se croisent ou se chevauchent, le novice et son tuteur officiel peuvent travailler sur des tâches et dans des lieux différents. Un agent de maintenance fait le constat suivant : "[J'étais tuteur d'un nouveau] Je n'ai pas eu beaucoup l'occasion de travailler avec lui, entre les formations... J'étais là, il n'était pas là. Je l'ai vu une journée [...]. Il sera avec moi une semaine pendant le mois. " (Laurent, 39 ans, chef d'équipe, 18 ans d'ancienneté et d'expérience en maintenance des installations)

Se met alors en place un tutorat collégial. Dans cette activité de maintenance qui peine à trouver des volontaires ${ }^{4}$, tous les membres du collectif de travail ont a priori intérêt à favoriser l'acquisition des savoirs et savoir-faire de métier chez leur collègue.

Cette forme de tutorat témoigne en creux du peu de cas réservé parfois à la transmission par tutorat. Son absence de programmation dans le cours des activités, au regard des ressources humaines disponibles, fait reposer la responsabilité de l'accompagnement des novices dans leurs apprentissages sur les équipes de travail qui se voient dans l'obligation d'assurer ce "maillage " dans un contexte de tension du marché interne d'emplois dans le secteur. Cette forme de tutorat ne peut qu'être informelle, les apprentissages se font de manière "opportuniste " (Gaudart et al., 2008) au gré des aléas, dans les interstices de l'action (Zara-Meylan \& Cau-Bareille, 2007). La présence, sur un îlot de quatre postes de gestionnaires, d'un agent de paie expert du domaine favorise la constitution d'un binôme informel apprenant-expert efficient dans la réalisation du travail, alors que la tâche de tutorat n’a pourtant pas été affectée à ce dernier.

4. Les conditions de travail, la mobilité géographique de plus en plus importante et l'intensification du travail de nuit sont autant de freins au recrutement d'agents de maintenance. 
Si l'intérêt des situations imprévues n'est pas remis en cause en termes de potentialité de développement des compétences dans le cours du travail, on ne peut en revanche ignorer les risques potentiels relatifs à la santé ou la sécurité des personnes et des installations concernées par ce type de situation, notamment si aucun lieu, ni aucun temps n'est réservé à la dimension tutorale. La gestion des aléas peut engendrer des débordements et une prise de risques non maitrisée qui nuit à la performance et à la préservation de la santé des tuteurs et/ou des apprenants (Delgoulet, 2012). Ainsi, le raccourcissement inopiné du délai de réalisation d'une tâche de maintenance, pour rendre à la production le système en état de fonctionnement, amène un opérateur-tuteur à prendre en charge sa tâche et celle de l'apprenant au risque de se blesser dans la précipitation.

Dans des versions plus formelles, ou moins improvisées, par exemple quand le responsable de service définit les objectifs et les personnes concernées par la tâche de tutorat, le tutorat collectif peut adopter un mode " tournant ", plusieurs membres du collectif devenant tuteur à tour de rôle. Dans le cadre de l'étude menée au sein d'équipes de gestionnaires de paie, ce type d'organisation du tutorat était mis en œuvre. Trois équipes ont été observées et seule l'une d'entre elles organisait le tutorat de la sorte. Dans ces services, chaque gestionnaire gère un portefeuille d'environ 300 dossiers de salariés. L'arrivée d'une nouvelle recrue peut être due au départ d'un gestionnaire ou à une augmentation du nombre de dossiers à gérer.

Dans le premier cas, le tutorat se fera en binôme avec l'agent sur le départ, comme l'explique Céline, gestionnaire de paie récemment recrutée à ce poste : "Tout de suite j’ai eu mon portefeuille avec mon binôme donc deux postes à gérer [...]. Lui, il n'avait pas envie de me former, il était en fin de carrière. Le peu quil mia formé, il l'a très bien fait " (Céline, 39 ans, gestionnaire de paie, 4 ans d'ancienneté, un an d'expérience en gestion administrative). On peut le constater ici, l'organisation en binôme fixe n'assure pas toujours un investissement du tuteur dans cette activité de transmission.

Dans le second cas en revanche, l'augmentation du nombre de dossiers nécessite un redécoupage des portefeuilles des gestionnaires. La responsable de l'équipe prend alors le temps d'organiser un tutorat " tournant " avant de procéder au redécoupage. Ce mode de tutorat permet de ne pas faire peser sur une seule personne l'activité de tuteur, ce qui représente une surcharge de travail puisque les tuteurs ne sont pas délestés d'une partie de leurs tâches pour assurer l'accompagnement et la transmission au novice des savoirs et savoir-faire. " $L a$ nouvelle en plus [de l'effectif prescrit pour l'équipe], au début : e-Learning pour apprendre le RH. Maintenant elle tourne avec chaque gestionnaire qui la prend en double pour apprendre un thème. [...] Après le tour fini, elle ira sur une table et, en fonction des affinités, elle posera les questions. Là, elle vient en plus alors que si c'était un gestionnaire qui partait, elle l'aurait formée avant de partir " (Brigitte, 43 ans, manager de proximité, équipe gestionnaires de paie, 20 ans d'ancienneté, 7 ans d'expérience en gestion administrative). 
On retrouve ici le poids déterminant de la définition des effectifs de travail sur les possibilités de mise en place d'une activité tutorale (Gaudart et al., 2008) : organiser une période de tutorat lorsque la personne nouvellement recrutée est en sureffectif (comme dans cette situation) permet de planifier davantage la contribution des tuteurs à la formation du novice. Toutefois, si cette organisation de l'activité tutorale est légitimée du fait qu'elle est mise en place par la responsable hiérarchique, les gestionnaires expérimentés qui encadrent à tour de rôle n'ont pas pour autant un statut de tuteur officiel. De ce fait, leur travail de professionnalisation n'est pas reconnu institutionnellement.

Cette mise en perspective des études réalisées au sein de cette grande entreprise confirme des travaux précédents sur le caractère souvent "improvisé » de la mise en place effective du tutorat (Delgoulet, 2012), parfois peu favorable au développement des compétences des tuteurs et apprenants. Elle dessine par ailleurs différents visages du tutorat, dont certains n'existent que localement et ne sont pas reconnus institutionnellement. Dès lors, l'évaluation des coûts ou gains économiques induits par le déploiement des tutorats est complexe, alors que certaines entreprises, soucieuses de gérer au plus près les investissements consacrés à la formation professionnelle, souhaitent en produire une mesure pour guider leurs choix futurs en la matière.

\section{Coûts et bénéfices du tutorat: des effets partagés}

Dans la plupart des cas, les bénéfices induits par le tutorat pour les salariés (développement d'une posture réflexive sur l'activité ; ré-enchantement du travail) profitent également à l'entreprise. A l'inverse, lorsque certaines situations tutorales " coûtent " au salarié, par surcharge de travail par exemple, elles peuvent pénaliser l'entreprise en retour : moindre investissement, posture de retrait, voire absentéisme. Il est ainsi bien délicat de spécifier systématiquement qui de l'entreprise ou des salariés est le plus directement concerné par les coûts et les gains inhérents aux diverses situations tutorales. L'hypothèse retenue ici est celle d'effets partagés entre l'entreprise, les tuteurs et les apprenants.

\subsection{Quels coûts ou bénéfices directs ?}

Parmi les coûts et bénéfices directs des tutorats, sont ici identifiées les dimensions relatives au temps consacré au tutorat et à la formation des tuteurs, d'une part ; aux qualités de tels dispositifs par rapport à des formations " classiques ", d'autre part.

\subsubsection{Des coûts directs : temps consacré au tutorat et formation des tuteurs}

Dans une première approche, le calcul des coûts directs générés par le tutorat nécessite, entre autres, de comptabiliser les ressources consommées dans la situation tutorale. Ces 
ressources sont majoritairement des heures consacrées par les salariés à leur rôle de tuteur. Néanmoins, identifier les tuteurs et repérer les situations tutorales est complexe, et isoler ce temps de tutorat n'a rien d'évident.

Lorsqu'il n'est pas attribué de temps spécifique à l'activité tutorale, celle-ci se réalise pour partie dans les creux de l'activité professionnelle : moments d'attente sur les chantiers, temps de trajet, déjeuner. De la même manière, le temps consacré au tutorat se fait souvent en débordement des horaires de travail pour les salariés soumis à des horaires stricts. Le tutorat peut aussi être totalement intriqué dans l'activité professionnelle. Pour un grand nombre de tuteurs, comme c'est le cas des managers encadrant un apprenti dans le cadre de cursus universitaires, il n'y a pas de temps fixe consacré au tutorat. Si l'investissement personnel dépend bien entendu des tuteurs eux-mêmes, pour certains tuteurs volontaires, ce travail de transmission et d'intégration est une mission voire une passion. Ils disent ainsi ne pas compter leurs heures et y consacrent "le temps qu'il faut" (Reyre et al., 2011).

Pour ces raisons, le temps de l'activité tutorale n'est pas quantifiable dans la plupart des configurations. Lorsque le tutorat est institutionnalisé, les tuteurs disposent alors d'un nombre d'heures officiellement dédiées à l'activité d'encadrement d'une nouvelle recrue ou d'un jeune en apprentissage. Cependant, même dans ces situations officialisant et légitimant l'activité, certains tuteurs ne déclarent pas le temps passé à l'activité tutorale et préfèrent imputer leurs heures sur des activités à visée directement productive pour l'entreprise. Dans un contexte organisationnel et économique qui vise à augmenter la productivité, le temps dévolu au tutorat est considéré de manière négative par certains managers; ils estiment en effet que le tutorat contribue avant tout à accroitre le ratio d'heures jugées non productives (Reyre et al., 2011).

Autre coût à considérer, celui que représente la formation des tuteurs. L'expertise technique ne suffit pas pour assurer une mission tutorale et bien faire son travail n'est pas toujours un gage de bien savoir en expliquer et transmettre tous les ressorts (SixTouchard, 1998). L'entreprise met en place des formations qui visent à fournir aux tuteurs quelques outils pédagogiques et à les sensibiliser aux enjeux économiques et aux transformations que connaît le secteur du transport et de la mobilité. Cependant, là encore, ces formations ne concernent pas tous les salariés qui prennent part à des situations tutorales, loin s'en faut. Par exemple, dans le cas des gestionnaires, tous les agents expérimentés ont eu, à un moment ou un autre, un rôle de tuteur mais aucun d'entre d'eux n'a bénéficié d'une formation spécifique. Le coût de ces formations ne reflète pas la réalité de ce qu'est le tutorat dans l'entreprise et se trouve être largement en-deçà de ce que pourraient coûter les formations de l'ensemble des tuteurs qui œuvrent informellement au sein de leurs entités. Le caractère informel de ces situations masque des besoins potentiels en formation des tuteurs, voire ne permet pas aux responsables de service de justifier des investissements dans de telles formations. 


\subsubsection{Des bénéfices directs : un dispositif flexible et moins dispendieux que les formations classiques}

Du côté des bénéfices pour l'entreprise, la Direction des Ressources Humaines de l'entreprise fait l'hypothèse que le tutorat est moins dispendieux qu'une formation en salle puisqu'il nécessite moins de ressources ${ }^{5}$. Si l'on se centre sur les coûts d'infrastructure et de matériels, il s'avère effectivement que la formule " tutorat " n'a pas besoin de salle, d'outils permettant de simuler le travail, de supports pédagogiques spécifiques, de formateur(s), etc. Au regard de la formation en salle, les situations tutorales sont surtout bien plus souples dans leur mise en ouvre (il n'est pas nécessaire d'attendre d'avoir suffisamment d'inscrits pour débuter) et plus " efficaces ". Elles permettent en effet à la nouvelle recrue de se confronter directement au réel pour y construire des connaissances "sur mesure "; un aspect souvent reproché aux formations plus classiques (Delgoulet, 2001 ; Chassaing, 2010) et que l'on retrouve dans les entretiens menés au sein de l'entreprise : "Il y a des formations mais la théorie et la réalité ça n’a rien à voir, ça donne une approche sur les textes réglementaires, etc. Les formations en salle avec un formateur, ça dépend, ça va d'une demi journée à trois jours. Il y en a, c'est que de la théorie; d'autres, le cas concret tu ne l'as pas en formation [...] Y a des thèmes que je vais voir là en formation et que je vais traiter que dans un an ", (Emile, 40 ans, gestionnaire de paie, 13 ans d'expérience en gestion administrative).

\subsection{Quels coûts et bénéfices indirects ?}

Bien sûr, les coûts et bénéfices du tutorat ne sont pas tous directs. Son intrication forte avec le travail multiplie les interrelations et, de fait, les coûts ou bénéfices indirects.

\subsubsection{Les coûts indirects : entre risques d'usure et moindre investissement des tuteurs}

L'activité tutorale peut être source de démotivation liée à une usure dans la répétition sans retour. Démotivation accrue si l'interprétation des éventuels échecs des actions d'accueil ou de tutorat renvoie, comme c'est souvent le cas, uniquement aux caractéristiques psychologiques et aux comportements sociaux des protagonistes : absence d'attitude collaborative, manque de courage, de respect de la hiérarchie (Gaudart et al., 2008). Les échecs sont alors personnalisés, les différences entre tuteurs et formés sont naturalisées : d'une part, les novices (souvent jeunes) ne voudraient pas apprendre, ne s'investiraient pas suffisamment dans le travail ; d'autre part, les expérimentés (âgés) ne voudraient pas transmettre. Les problèmes sont ainsi externalisés et l'on invoque l'évolution de la Société et des valeurs en général, sans connaître vraiment ce à quoi elles faisaient référence auparavant (Rousselet, 1987). Or, sans nier la part d'intersubjectivité, ces difficultés sont révélatrices, dans bien des cas, de conflits entre plusieurs logiques de gestion des ressources humaines,

5. Hypothèse dont il est difficile de confirmer la validité étant donné la complexité de l'évaluation des coûts et des gains dont cet article se fait l'écho. 
voire entre ces logiques et celles de production ou de service. Les entreprises demandent aux nouveaux et anciens de coopérer, de s'entraider, d'échanger des informations comme si les changements apportés par les dispositifs de $\mathrm{GRH}^{6}$ n'avaient pas d'incidence sur les possibilités réelles de coopérer, de s'entraider et de transmettre. Comme si l'utilisation conjointe de ces dispositifs sous-tendant des logiques d'actions antagonistes (embauche par intérim et dispositif de fidélisation par exemple) ne venait pas compromettre, dans le travail, toute coopération ou efficience de la transmission.

Cette usure des tuteurs peut aussi être liée à la "pénibilité perçue " de ces situations (Molinié \& Volkoff, 2006). C'est ce que nous avons constaté dans certaines situations où il y a un flux important de nouvelles recrues, à cause d'un taux élevé de turnover ou d'une restructuration. Dans ces conditions, l'activité tutorale peut être très stratégique dans une optique de professionnalisation et chronophage pour les salariés qui s’y investissent. La sur-sollicitation des salariés expérimentés engendre une "lassitude de l'acteur" (Alter, 1993), d'autant plus lorsque l'activité tutorale est informelle et basée sur le volontariat.

Une gestionnaire expérimentée parle de cette usure : "J'ai toujours quelqu'un de nouveau en face de moi. Avant Michèle, j’avais une autre Michèle et là Laurence, avant Bénédicte, elles sont en face de moi donc sans arrêt elles me posent des questions. [...] Niveau formation, ça devient de plus en plus crispant. C'est pénible, on estime que la personne qui arrive elle devrait avoir vu le fonctionnement du micro, le logiciel [de paie]. On est ralenti et on l'a fait X fois, c'est pénible, on n'est pas payé pour ça». (Patricia, 51 ans, gestionnaire de paie, 30 d'ancienneté, 19 ans d'expérience en gestion administrative). Marianne, autre collègue expérimentée, confirme : "Les arrivées, départs c'est pas facile, ça a des effets sur les portefeuilles. Et puis ce n'est pas facile de travailler dans une équipe pas stable. Il faut se replonger dans les dossiers et quand on ne comprend pas trop ce que l'autre a fait. [...] Faut toujours former la personne qui arrive et aujourd'hui il n'y a plus que ça, des gens en reconversion " (Marianne, 50 ans, gestionnaire de paie, 30 ans d'ancienneté et d'expérience en gestion administrative). Cette usure génère un moindre engagement dans l'activité tutorale, qui peut aller jusqu'au refus (Gaudart et al., 2008). Dans le cas des gestionnaires, l'analyse des entretiens révèle que cette usure est un des éléments à l'origine de l'augmentation de l'absentéisme chez les salariés expérimentés, ce que confirme un responsable : "L'absentéisme émane certainement du fait que l'on a épuisé nos troupes à faire des remplacements haut le pied sans renfort supplémentaire [...] Paradoxalement, on a plus d'absentéisme de [salariés] expérimentés que les autres. " (Miguel, 45 ans, responsable des équipes de gestionnaires de paie) Il s'agit bien là d'un coût indirect tant pour les salariés que pour l'entreprise dont il faudrait pouvoir tenir compte.

6. La multiplication de l'intérim et de la sous-traitance, la diversification des offres de formation, les dispositifs de fidélisation, l'évaluation individuelle des performances, la gestion du personnel par les compétences, etc. 


\subsubsection{Des gains indirects : entre prévention des risques professionnels et maintien en emploi}

Ce qui se transmet va bien au-delà des seuls savoirs techniques : "Tout s'apprend sur le tas ici. C'est du relationnel, c'est du management, de la technique dans certains cas, de la diplomatie. C'est beaucoup de choses. " (David, 27 ans, 8 ans d'ancienneté et d'expérience dans la maintenance des installations). Dans de nombreux cas, ces situations tutorales peuvent donner à voir des stratégies de préservation de sa santé, très utiles dans le travail, et qui ne sont pas enseignées dans les formations classiques (Gaudart, 2003 ; Cloutier et al., 2002). Toutefois, des choix organisationnels peuvent venir faire obstacle, comme le regrette cet agent: "Les jeunes n'ont pas la formation d'avant. Déjà, on a moins de temps à leur consacrer. Avant, on pouvait se permettre, quand on était plus nombreux, de prendre des jeunes avec nous, de leur dire: "Tu vois. On va passer une corne comme ça". C'est très important même si ça paraît rien du tout. "On porte une échelle comme ça". C'est très important de porter une échelle comme ça, sinon on se fait mal. » [...] "Nous n'avons plus le temps d'y porter attention " (Roger, 51 ans, adjoint du responsable de brigade, 32 ans d'ancienneté et d'expérience dans la maintenance des installations). Ces stratégies sont pourtant de potentielles ressources pour une prévention durable des risques professionnels, en permettant de diminuer les dépenses en matière de maladies professionnelles et d'accidents du travail. Il s'agit bien là de bénéfices indirects pour l'entreprise induits par des bénéfices indirects pour les salariés.

Plus largement, l'activité tutorale a d'autres effets d'ordre subjectif chez les tuteurs et les apprentis. Elle est source de motivation lorsque, à travers l'action tutorale, l'agent trouve un intérêt renouvelé pour son travail. Le tutorat est ici l'occasion de valoriser l'expérience des "anciens " et de mutualiser des pratiques réflexives avec les " nouveaux " qu'ils accueillent, voire entre les anciens eux-mêmes (Thébault et al., 2012). C'est ce qui conduit certains agents de maintenance à assumer le rôle de tuteur sans en avoir le statut : la satisfaction qu'ils trouvent dans la transmission de leurs acquis leur permet de passer outre le fait que l'entreprise leur refuse un statut ouvrant droit à compensation financière. Cette implication des salariés expérimentés peut ouvrir sur de réelles possibilités de reconversion en deuxième partie de carrière, où la fonction de formateur prendra une place croissante (Wittorski, 1996). L'entreprise peut donc y voir un moyen de répondre partiellement aux enjeux économiques et sociaux du maintien en emploi des salariés les plus âgés dans une perspective d'allongement des carrières?

Ces aspects (organisationnels, de santé, de reconnaissance au travail), décisifs dans l'efficience du tutorat, sont très difficilement quantifiables. On se situe ici sur le versant des coûts cachés qui résultent, pour partie, de la faible contractualisation du tutorat dans les entreprises. Ainsi, il nous semble qu'une approche uniquement quantitative,

7. Bien qu'il faille se méfier du lien faussement évident seniors/tuteurs (Masingue, 2009). 
en termes de coûts et de gains, n'est pas pertinente et ne saurait faire l'impasse sur le volet qualitatif.

\section{Conclusion : mesurer ou prendre la mesure ?}

Le développement non démenti du tutorat en entreprise, depuis plusieurs dizaines d'années ainsi que la diversité de ses fonctions et modalités de mise en œuvre conduisent aujourd'hui certaines entreprises à s'interroger sur l'investissement financier et humain que sous-tend un tel dispositif de formation. Dans un souci de maîtrise, voire de réduction du financement de la formation professionnelle, un des enjeux pour les responsables des ressources humaines et les partenaires sociaux est de cerner les coûts et bénéfices des tutorats actuellement dispensés. Un récent rapport du Conseil National pour la Formation Professionnelle Tout au Long de la Vie (CNFPTLV, 2013), consacré exclusivement à l'évaluation du coût de l'apprenti (en formation par alternance), confirme l'intérêt que peut représenter cette connaissance pour les acteurs de l'apprentissage (État, régions, CFA - Centre de formation d'apprentis - ou entreprises) ; cependant, il souligne aussi les difficultés à considérer l'ensemble des déterminants économiques du " coût complet " ${ }^{8}$ de formation par apprenti, alors que cette situation de formation est pourtant bien cadrée et qu'il n'est pas envisagé de pousser l'analyse au-delà des dépenses financières directes et effectives.

Dans ce contexte, la synthèse de travaux menée ici montre qu'au-delà des coûts et bénéfices directs qui ne sont pas à négliger, l'étude des conditions concrètes d'exercice du tutorat permet de mettre en évidence les coûts et les gains cachés (notamment coût sous-estimé de la formation des tuteurs; usure des tuteurs dans la répétition des situations tutorales; prise de risque accrue dans la gestion des aléas ; corollaires de l'absence de reconnaissance du travail supplémentaire fourni ; etc.), liés notamment à des objectifs non fixés par l'institution (des situations tutorales informelles tenant sur la disponibilité effective ou contrainte des plus anciens), voire contradictoires (désignation de salariés ayant la fonction de tuteur sans reconnaître le travail supplémentaire que cette tâche suppose ; ajustement des effectifs au plus juste et accueil des novices dans le cadre de tutorats, etc.).

Cette approche de la mesure du tutorat ne fournit pas de solution prête à l'emploi sur la mesure. Elle a cependant le mérite de pointer la complexité de la tâche probablement sousestimée par les commanditaires dans l'entreprise étudiée. On comprend ici que les coûts et bénéfices du tutorat ne peuvent être considérés uniquement depuis le versant financier et gestionnaire stricto sensu, contrairement aux attentes de certains interlocuteurs de la Direction des Ressources Humaines, porteurs de la demande initiale d'évaluation des coûts du tutorat.

8. Le CNFPTLV définit le "coût complet» de formation par apprenti comme le coût comptable corrigé des produits en atténuation de charges (par mutualisation ou répercussion d'une partie des charges sur une autre structure), des charges exceptionnelles et charges supplétives (coût des locaux, des personnels sur le budjet de l'Etat, des moyens techniques de formation). 
On rejoint ici le constat de la contingence du tutorat par rapport au travail (Barbier, 1996) : dans son organisation et la progression dans les apprentissages, ou ses moyens matériels et humains notamment. Ainsi, de notre point de vue, les coûts et les gains doivent également être pensés sous l'angle des relations sociales, de l'organisation du travail et de ses exigences de production de biens ou de services, du développement des compétences et de la santé que le tutorat télescope dans son articulation au travail. Cette approche montre aussi le nécessaire passage par l'analyse de l'activité tutorale en situation qui permet d'appréhender la diversité des formes de tutorat, les relations qui se nouent entre les différentes dimensions citées ci-dessus et leurs conséquences sur le développement des formés, mais aussi des tuteurs, leur efficience au travail et leur santé.

L'ampleur de la tâche et de ses ramifications confère, dans ces conditions, une dimension herculéenne à la mesure du tutorat, comme ont pu le conclure Reyre et ses collègues (2011). Mesurer de manière plus pertinente supposerait que l'on fournisse un cadre à cette mesure et que l'on définisse en amont a minima:

- le cadre légal dans lequel se déploie le tutorat : par exemple, fait-il l'objet d'un cadre juridique et financier précis, comme celui du contrat d'apprentissage, ou bien relève t-il de l'informel ?

- les termes de sa dimension normative : est-il associé à une promesse de qualification du formé, de rétribution du tuteur ? Se déroule-t-il en binôme ou au sein d'un collectif ? Est-il encadré par une charte?

- les objectifs qui lui sont assignés ou les effets attendus : est-il un outil de socialisation qui permet d'accueillir et fidéliser les nouvelles recrues, de fédérer des collectifs de travail autour d'un genre professionnel (Clot et al., 2007) ? Est-il un dispositif pédagogique voué à la transmission de savoirs et savoir-faire techniques mais aussi de stratégies de préservation de sa santé, d'échanges réciproques favorisant la co-construction des savoirs, la créativité entre générations de travailleurs ? Est-il un outil de réduction des investissements en formation, ou encore de maintien en emploi des seniors ? Est-il une modalité de transformation de l'entreprise (Wittorski, 1996) ? Est-il une combinaison de plusieurs objectifs ?

- l'adéquation entre les moyens organisationnels et règlementaires, techniques, pédagogiques, humains mis en place, les objectifs fixés dans le cadre d'un dispositif tutoral et les résultats constatés en termes de développement effectif des compétences professionnelles.

Il s'agira alors de confronter le cadre prescrit du tutorat à la réalité de l'activité tutorale et de caractériser les écarts (par exemple dans la veine des travaux menés en ergonomie). Toutefois, les formes de tutorat ne se prêtent pas toutes a priori à la formalisation. Comme nous avons pu l'observer chez les gestionnaires de paie, l'identité de tuteur et la pratique du tutorat sont à faire reconnaître, à inventer, à construire. En l'absence de ce cadre, il n'est alors pas question de "mesurer " mais, en premier lieu, de se donner les moyens d'identifier les représentations, la manière dont chaque tuteur invente son rôle, sa pratique pédagogique et ses responsabilités. 
Par ailleurs, en reprenant les travaux menés sur la mesure du travail dans ses relations à la santé des travailleurs et travailleuses (Gollac \& Volkoff, 2010), il semble utile tout d'abord de statuer sur les objectifs des dispositifs de mesure. Sont-ils pensés en tant qu'évaluation éclairant et orientant les décisions d'une entreprise ou en tant qu'état des lieux fournissant des connaissances générales et permettant de sensibiliser les acteurs à la question du tutorat? Il convient également de ne pas se méprendre sur les qualités de la mesure, a fortiori quantitative, qui, plus que "la vérité » des faits expertisés, devrait s'attarder sur "l'authenticité " des situations vécues (Volkoff, 2005). Par ailleurs, il semble plus pertinent d'envisager la mesure au pluriel, dans une approche multidimensionnelle, pour éviter les interprétations simples de causes à effets peu pertinentes dans l'analyse du travail (ici de tutorat) et son évaluation. Enfin, ces mesures ont d'autant plus d'intérêt qu'elles sont réitérées dans le temps afin de sonder les évolutions.

Compte tenu de ces éléments, et à condition que le tutorat bénéficie d'un cadre défini, la mesure de ses coûts et bénéfices dans ses diverses formes pourrait passer par la construction d'une enquête ou d'un questionnaire issu d'une analyse préalable de l'activité tutorale par observations et entretiens (Prunier-Poulmaire \& Gadbois, 2005). Cette méthodologie permettrait de recueillir des points de vue différenciés (des tuteurs, des formés, des collègues, des supérieurs hiérarchiques, des acteurs de la filière ressources humaines, etc.) sur l'activité tutorale vécue et ses conséquences diverses et variées. Elle représenterait une opportunité de produire des mesures contrastées sur un même objet et qui pourraient le cas échéant être répétées.

La multiplicité des situations tutorales, de leurs formes, des fonctions qui leur sont confiées dans des conditions réelles de réalisation plus ou moins favorables, de même que les difficultés d'identification du temps que consacrent les tuteurs à cette tâche en parallèle de leur travail de production de biens ou de services compliquent leur "simple " mesure économique. Les difficultés associées à la mesure du tutorat dans l'entreprise sont légion; nous avons participé à leur identification. Ceci ne signifie pas qu'elles sont insurmontables, à condition que le cadre de la mesure soit établi et que l'approche quantitative soit complétée d'un versant qualitatif déployé selon une démarche évaluatrice intégrative ; cette démarche associe à l'évaluation des coûts et avantages d'un dispositif de formation par rapport à d'autres (tutorat $v$ formation classique en salle ou selon un format de e-learning), une évaluation de l'adéquation des méthodes pédagogiques et des moyens mis à disposition, compte tenu des objectifs d'apprentissage définis par les prescripteurs du tutorat ou, à défaut, inférés de la tâche à laquelle on forme (Berthelette et al., 2006).

9. Dans l'ouvrage "L'ergonomie et les chiffres de la santé au travail: ressources, tensions et pièges", Serge Volkoff insiste sur la place qui doit être faite au point de vue des travailleurs dans la mesure du travail, de la santé au travail et des relations santé-travail. Il pointe notamment l'importance d'intégrer dans les démarches de mesure quantitative, du travail ou de la santé, la subjectivité des personnes qui vivent ces situations afin d'en restituer les particularités. 
Alter N. (1993), «La lassitude de l'acteur de l'innovation », Sociologie du travail, vol. 35, n 4, pp. 447-468.

Barbier J.-M. (1996), «Tutorat et fonction tutorale : quelques entrées d'analyse ", Recherche et Formation, 22, pp. 7-19.

Berthelette D., Leduc N., Bilodeau H., Durand M.J., Faye C., Loignon C. \& Lagac M.C. (2006), Analyse d'un programme de formation visant la prévention primaire des maux de dos chez le personnel soignant des centres hospitaliers au Québec, Rapport n 452, Montréal : IRSST.

Boru J.-J. \& Leborgne C. (1992), Vers l'entreprise tutrice, Paris : Editions Entente.

Chassaing K. (2010), «Les "gestuelles” à l'épreuve de l'organisation du travail : du contexte de l'industrie automobile à celui du génie civil ", Le travail humain, 73(2), pp. 163-192.

Clot Y., Fernandez G. \& Scheller L. (2007), « Le geste de métier : problèmes de la transmission ", Psychologie de l'interaction, 23-24, pp. 109-138.

Clot Y. (2001), "Méthodologies en clinique de l'activité. L'exemple du sosie ", in Santiago M., Delefosse \& Rouan G. (Eds), Les méthodes qualitatives en psychologie Paris : Dunod, pp. 125-147.

Cloutier E., Lefebvre S., Ledoux E., Chatigny C. \& St-Jacques Y. (2002), Enjeux de santé et de sécurité au travail dans la transmission des savoirs professionnels: le cas des usineurs et des cuisiniers, Rapport n ${ }^{\circ}$ 316, Montréal : IRSST.

Conseil économique et social (2006), Plan national d'action concerté pour l'emploi des seniors 2006-2010. Conférence nationale sur l'emploi des seniors, le 6 juin, Paris. http:// www.travail-emploi-sante.gouv.fr/ses-actions,277/plan-national-d-action-concerte,542/ plan-national-d-action-concerte,3428.html

Conseil National pour la Formation Professionnelle Tout au Long de la Vie (2013), Le coût par apprenti. Aspects méthodologiques, Rapport final, le 13 octobre, Paris. http:// www.cariforef-pdl.org/telechargement/ficTelecharge_1/Documentation/GED_lettres_ dinfo/GED_EnBref/2013COUTAPPRENTI.pdf

Delgoulet C. (2001), « La construction des liens entre situations de travail et situations d'apprentissage dans la formation professionnelle ", Pistes, vol. 3, n 2.

Delgoulet C. (2012), «Apprendre pour et par le travail : les conditions de formation tout au long de la vie professionnelle ", in Gaudart C., Molinié A. \& Pueyo V. (Eds.), La vie professionnelle : âge, expérience et santé à l'épreuve des conditions de travail, Toulouse : Octarès, pp. 46-74. 
Fredy-Planchot A. (2007), "Reconnaître le tutorat en entreprise ", Revue Française de Gestion, n 175, pp. 23-32.

Gaudart C. (2003), «La baisse de la polyvalence avec l'âge : question de vieillissement, d'expérience, de génération ? ", Pistes, 5(2), pp. 1-25.

Gaudart C., Delgoulet C. \& Chassaing K. (2008), « La fidélisation de nouveaux dans une entreprise du BTP : Approche ergonomique des enjeux et des déterminants ", Activités, 5(2), 2-24. http://www.activites.org/ v5n2/v5n2.pdf

Gollac M. \& Volkoff S. (2010), « Mesurer le travail. Une contribution à l'histoire des enquêtes françaises dans ce domaine ", Document de travail n 127 , juillet, CEE.

Jolivet A., Delgoulet C. \& Volkoff S. (2009), Étude exploratoire Age Santé travail: Pénibilité et gestion des parcours professionnels à la SNCF, Rapport final, Juin.

Kloetzer L. (2011), « L'engagement conjoint dans la pratique comme clef du développement de l'activité des tuteurs ", ePTO, 17, pp. 445-464.

Largier A. \& Tirilly G. (2011), «Innovations socio-organisationnelles et collectifs de gestionnaires de paie ", IVème congrès de l'Association Française de Sociologie, Grenoble.

Masingue B. (2009), Seniors tuteurs : comment faire mieux ? Rapport au secrétaire d'état chargé de l'emploi. http://www.ladocumentationfrancaise.fr/var/storage/rapportspublics/094000125/0000.pdf

Miles B. \& Huberman A.-M. (2003), Analyse des données qualitatives, Bruxelles : De Boeck Université.

Molinié A.F. \& Volkoff S. (2006), «Fin de vie active et pénibilités du travail », in L'Horty Y. (Ed.), La qualité de l'emploi, pp. 95-104, Paris : La Découverte.

Oddone I. (1981), Redécouvrir l'expérience ouvrière. Vers une autre psychologie du travail ? Paris : Editions sociales.

Prunier-Poulmaire S. \& Gadbois C. (2005), "Quand le questionnaire s'impose à l'ergonome ", in Volkoff S. (Ed.), L'ergonomie et les chiffres de la santé au travail : ressources, tensions et pièges, pp. 76-86, Toulouse : Octares.

Reyre G., Zarifian P., Iazykoff V., Kloetzer L. et Werthe C. (2011), Pratiques de tutorat et liens intergénérationnels, Rapport SNCF.

Rousselet J. (1987), "Attitudes des jeunes face au travail et au non travail ", in LévyLeboyer C. \& Sperandio J.-C. (Eds), Traité de psychologie du travail, pp. 57-76, Paris : PUF.

Six-Touchard B. (1998), "Développement de la compétence tutorale par l'auto-analyse du travail », Éducation Permanente, 138(2), pp. 87-98. 
Thébault J., Gaudart C., Cloutier E. \& Volkoff S. (2012), "Transmission of vocational skills between experienced and new hospital workers”, Work, 41(2), pp. 195-204.

Volkoff S. (Ed) (2005), L'ergonomie et les chiffres de la santé au travail : ressources, tensions et pièges, Toulouse : Octarès.

Wittorski R. (1996), "Évolution des compétences professionnelles des tuteurs par l'exercice du tutorat ", Recherche et Formation, 22, pp. 35-46.

Zara-Meylan V. \& Cau-Bareille D. (2007), " La transmission de la gestion des risques : le fruit d'une interaction singulière dans les interstices de l'activité de travail ", Journée $I M D R$, Paris, juin. 